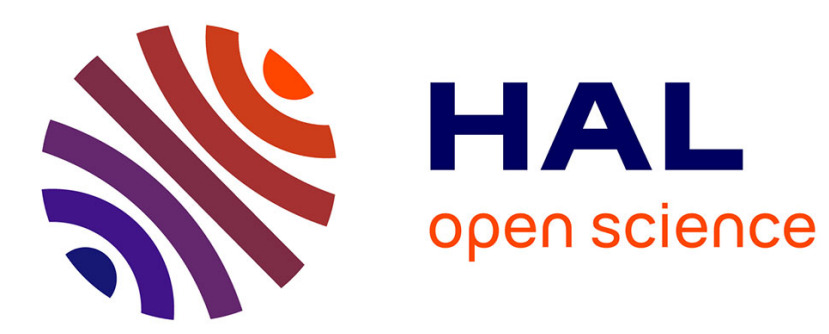

\title{
Ge-rich graded-index SiGe alloys: exploring a versatile platform for mid-IR photonics
}

J.M Ramirez, Q Liu, V Vakarin, J. Frigerio, A Ballabio, D Chrastina, X Le Roux, C Alonso-Ramos, G Isella, L Vivien, et al.

\section{- To cite this version:}

J.M Ramirez, Q Liu, V Vakarin, J. Frigerio, A Ballabio, et al.. Ge-rich graded-index SiGe alloys: exploring a versatile platform for mid-IR photonics. ICTON 2018, Jul 2018, Bucarest, Romania. hal-02362444

\section{HAL Id: hal-02362444 \\ https://hal.science/hal-02362444}

Submitted on 13 Nov 2019

HAL is a multi-disciplinary open access archive for the deposit and dissemination of scientific research documents, whether they are published or not. The documents may come from teaching and research institutions in France or abroad, or from public or private research centers.
L'archive ouverte pluridisciplinaire HAL, est destinée au dépôt et à la diffusion de documents scientifiques de niveau recherche, publiés ou non, émanant des établissements d'enseignement et de recherche français ou étrangers, des laboratoires publics ou privés. 


\title{
Ge-rich graded-index SiGe alloys: exploring a versatile platform for mid-IR photonics
}

\author{
J. M. Ramirez ${ }^{1 *}$, Q. Liu ${ }^{1}$, V. Vakarin ${ }^{1}$, J. Frigerio ${ }^{2}$, A. Ballabio ${ }^{2}$, D. Chrastina ${ }^{2}$, X. Le Roux ${ }^{1}$, C. Alonso- \\ Ramos $^{1}$, G. Isella ${ }^{2}$, L. Vivien ${ }^{1}$ and D. Marris-Morini ${ }^{1}$ \\ ${ }^{I}$ Centre de Nanosciences et de Nanotechnologies, Université Paris Sud, CNRS, Université Paris Saclay, 91405 \\ Orsay, France \\ ${ }^{2}$ L-NESS, Dipartimento di Fisica, Politecnico di Milano, Polo di Como, Via Anzani 42, 22100 Como, Italy \\ *e-mail: joan-manel.ramirez@u-psud.fr
}

\begin{abstract}
In this paper, the recent progress on a new Ge-rich SiGe platform for mid-IR integrated photonics is presented. Low-loss spiral waveguides working over a broadband wavelength range are discussed, followed by a sensing proof-of-concept using a standalone photoresist with a known spectral absorption pattern. In addition, the development of new mid-IR interferometric devices for wavelength filtering and enhancement of the lightmatter interaction are presented. Finally, efficient designs to exploit the third-order nonlinearities in these Gerich SiGe waveguides at mid-IR wavelengths are shown. The demonstration of these key building blocks will pave the way towards the implementation of new mid-IR photonic integrated systems with multiple
\end{abstract} functionalities.

Keywords: Mid-IR integrated photonics, SiGe alloys, mid-IR spectroscopy, waveguides, interferometric optical devices, non-linear mid-IR photonics.

\section{INTRODUCTION}

Mid-IR integrated photonics is recently gaining attention due to the wide range of applications foreseen including chemical and biological sensing, thermal imaging, early medical diagnosis or secure communications [1]. Among others, the possibility to use the mature CMOS technology to develop novel photonic integrated circuits exploiting the unique molecular mid-IR absorption patterns of several chemical and biological substances rises as one of the driving forces that push this research field. This approach would enable ultrasensitive label-free photonic integrated sensors which could be integrated along with other components in a single photonic chip, hence opening the route towards multifunctional photonic systems. For that, however, a suitable platform capable of integrating several photonic components such as waveguides, Bragg filters, resonators or Mach-Zehnder interferometers should be implemented. Promising mid-IR integrated platforms to carry out this task stand for $\mathrm{Ge}$-on $\mathrm{Si}$, suspended $\mathrm{Si}$ or SiGe alloys with tailored Ge concentration, among others [2-4]. This latter approach is expected to offer high versatility and broadband transparency [5], leading to the development of important photonic building blocks such as low-loss waveguides and wideband Mach-Zehnder interferometers for the mid infrared [6, 7]. In spite of the progress achieved so far using SiGe alloys, there are still several open questions that require further exploration, such as the disclosure of the platform transparency bandwidth, the sensitivity background or the investigation of the nonlinear properties in the mid-IR, taking advantage from the absence of two-photon absorption at early mid-IR wavelengths [8].

Thus, in this paper we will review our work on the development of a new Ge-rich SiGe platform for mid-IR photonics. Spiral waveguides showing broadband low-loss operation will be revisited, followed by the demonstration of a real sensing scenario based on molecular absorption within the evanescent field in waveguides cladded with a standalone photoresist. Additionally, the work done towards the demonstration of interferometric devices working at mid-IR wavelengths will also be shown. Finally, recent works on nonlinear active devices will be discussed, providing a comprehensive roadmap towards the exploitation of the third-order nonlinearity in Ge-rich SiGe systems to generate a supercontinuum at mid-IR wavelengths.

\section{BROADBAND LOW-LOSS GE-RICH SIGE WAVEGUIDES}

The development of photonic integrated structures in the mid-IR requires first a preliminary study to retrieve the best design parameters for efficient mode propagation over a wide range of wavelengths. In this regard, our design parameters have been optimized to cover a wavelength range from $\lambda=5.5 \mu \mathrm{m}$ to $8.5 \mu \mathrm{m}$, since this is the available wavelength range in our experimental setup. Furthermore, the material constraints should also be considered, since Ge and Si have different lattice parameters. As a consequence, the growth of Ge-rich layers on Si substrates can result in the generation of a highly defective layer-substrate interface which is lossy and therefore limits the overall performance for photonic applications. In order to palliate this circumstance, a graded buffer layer is grown first, with a gradual increase of the Ge concentration in the growth direction to accommodate the lattice mismatch between $\mathrm{Si}$ and $\mathrm{Ge}$, providing a linear increase of the vertical refractive index, as depicted in figure 1(a). Then, a Ge-rich SiGe layer is grown with a slightly higher Ge concentration, 
providing sufficient refractive index contrast to enable the confinement of an optical mode. By doing that, we obtain high quality layers with a low threading dislocation density (TDD) in the order of $3 \times 10^{6} \mathrm{~cm}^{-2}$. Then, a partial etching of the SiGe stack is used to define the rib-like waveguide structures, as seen in the schematic waveguide cross-section of figure 1(b). In our case, we have defined $4 \mu \mathrm{m}$-wide waveguides with an equally etching depth of $4 \mu \mathrm{m}$ since it provides a good trade-off between mode confinement and moderate sidewall roughness using the available fabrication facilities. A set of spiral waveguides with different lengths, i. e. $\mathrm{L}=0.6$ $\mathrm{cm} / 4.2 \mathrm{~cm} / 6.1 \mathrm{~cm} / 8.3 \mathrm{~cm}$, were fabricated to measure propagation losses by means of the cut-back technique in butt-coupling configuration [11]. Figure 1(c) shows a top microscope view of the fabricated spirals, along with an inset of a representative finite-difference-method (FDM) simulation of the propagating optical mode for an operating wavelength of $\lambda=5.8 \mu \mathrm{m}$ and a TE polarization. The measured propagation loss characteristic can be observed in figure $1(\mathrm{~d})$, showing a flat performance with losses comprised between $2-3 \mathrm{~dB} / \mathrm{cm}$ for both polarizations. This performance demonstrates the potential of these waveguides to be used as fundamental blocks for the development of mid-IR photonic integrated circuits at these wavelengths.
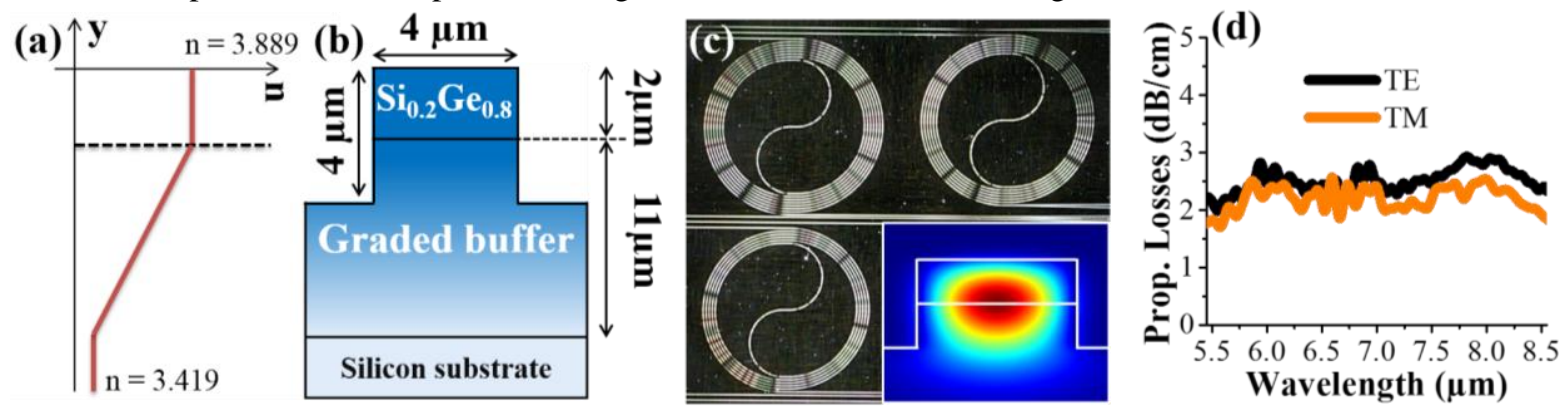

Figure 1: (a) Vertical refractive index profile of waveguides. (b) Waveguide cross-section. (c) Top view of fabricated waveguide spirals. Inset shows an FDM optical simulation of the propagating TE mode at $\lambda=5.8 \mu m$. (d) Measured propagation loss characteristic over the available wavelength range from $\lambda=5.5 \mu \mathrm{m}$ to $8.5 \mu \mathrm{m}$ for both TE (in black) and TM (in orange) polarizations.

\section{MID-IR SENSING USING AN ABSORPTIVE UPPER CLADDING}

The previous section has shown promising mid-IR transmission characteristics for Ge-rich SiGe spiral waveguides. Bearing in mind one of the main driving applications of these photonic integrated chips, which is the conception of new effective mid-IR sensing systems, a basic proof-of-concept was conceived to inspect the sensitivity of our waveguides to changes in the upper cladding [12]. For that, we spin-coated the spiral waveguides with a standard photoresist (S1818) and measured the changes in the transmission spectrum. Figure 2 shows representative SEM images of the waveguides before (a) and after (b) covering them with the photoresist. As a particular example, figure 2(c) displays the comparison between the transmission of an aircladded (black line) and a photoresist-cladded (orange line) waveguide with a total length of $\mathrm{L}=8.3 \mathrm{~cm}$. As can be observed, several absorption patterns appear in the photoresist-cladded waveguide uniquely, which can be associated to different molecular absorption bands of the photoresist. To corroborate this fact, the absorption spectrum of the photoresist was retrieved from transmission measurements in spiral waveguides over a wavelength range from $\lambda=5.2 \mu \mathrm{m}$ to $7 \mu \mathrm{m}$, obtaining a good correlation with the photoresist absorption spectrum retrieved when performing normal-incidence transmittance measurements in a standalone $\mathrm{Si}$ wafer spin-coated with the same photoresist. Figure 2(d) illustrates this comparison, showing a close correlation of the different absorption peaks. It should be noticed that the absorption spectra have been normalized and up-shifted for convenience.
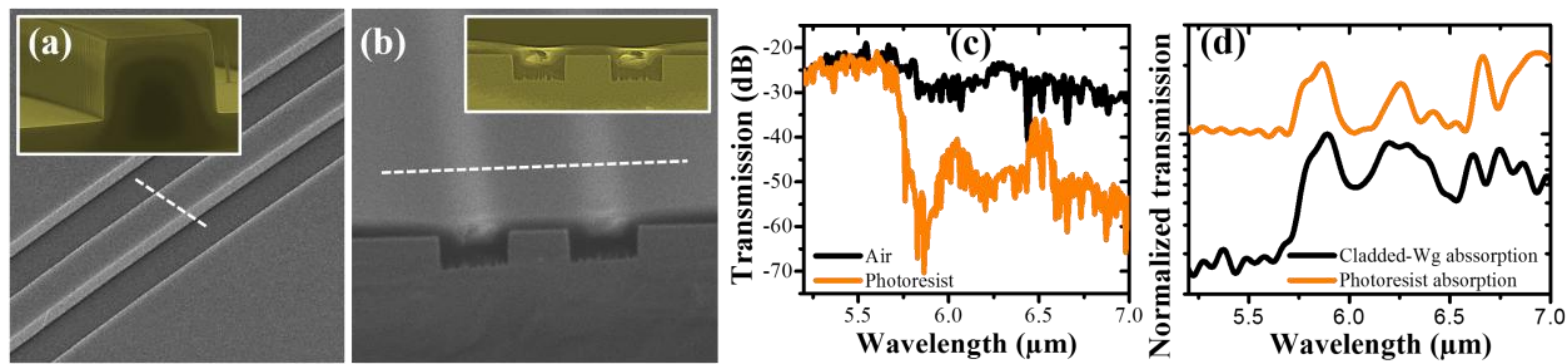

Figure 2. SEM image of the fabricated SiGe waveguide with an air cladding (a) and with an S1818 photoresist cladding (b). Insets show closer cross-section SEM views of the waveguide corresponding to the white-dashed line. (c) Mid-IR transmission for an air-cladded (in black) and a photoresist-cladded (in orange) spiral waveguide with a total length of $L=$ $8.3 \mathrm{~cm}$. (d) Comparison of the retrieved absorption pattern of the $\$ 1818$ photoresist obtained from transmission measurements in waveguides (in black) and the measured absorption spectrum of the photoresist obtained from surface illuminated experiments (in orange). 


\section{INTERFEROMETRIC DEVICES: MACH-ZEHNDER INTERFEROMETERS}

Aside from waveguides, the demonstration of integrated optical components able to deliver interferometric patterns in the mid-IR is also a major requirement to implement complex photonic circuits with manifold functionalities. In particular, the demonstration of Mach-Zehnder interferometers is interesting to perform multiple wavelength filtering and eventually mid-IR Fourier transform integrated spectrometers. With this aim, we recently demonstrated asymmetric Mach-Zehnder interferometers working over a broadband wavelength range from $\lambda=5.5 \mu \mathrm{m}$ to $8.5 \mu \mathrm{m}$ for both polarizations, showing good tunability of the free spectral range and an extinction ration of at least $10 \mathrm{~dB}$ regardless of the operation wavelength range [7]. Figure 3(a) displays a top view of the fabricated devices, along with a SEM view of the multimode interferometer responsible for the power splitting and merging of the propagating optical mode. Figure 3(b) describes a representative spectrum of a Mach-Zehnder device containing a difference arm length of $\Delta \mathrm{L}=48 \mu \mathrm{m}$ working all over the available wavelength range in the mid-IR. Thus, good prospects for the development of new Fourier transform integrated spectrometers are envisioned by combining these fundamental interferometric building blocks.
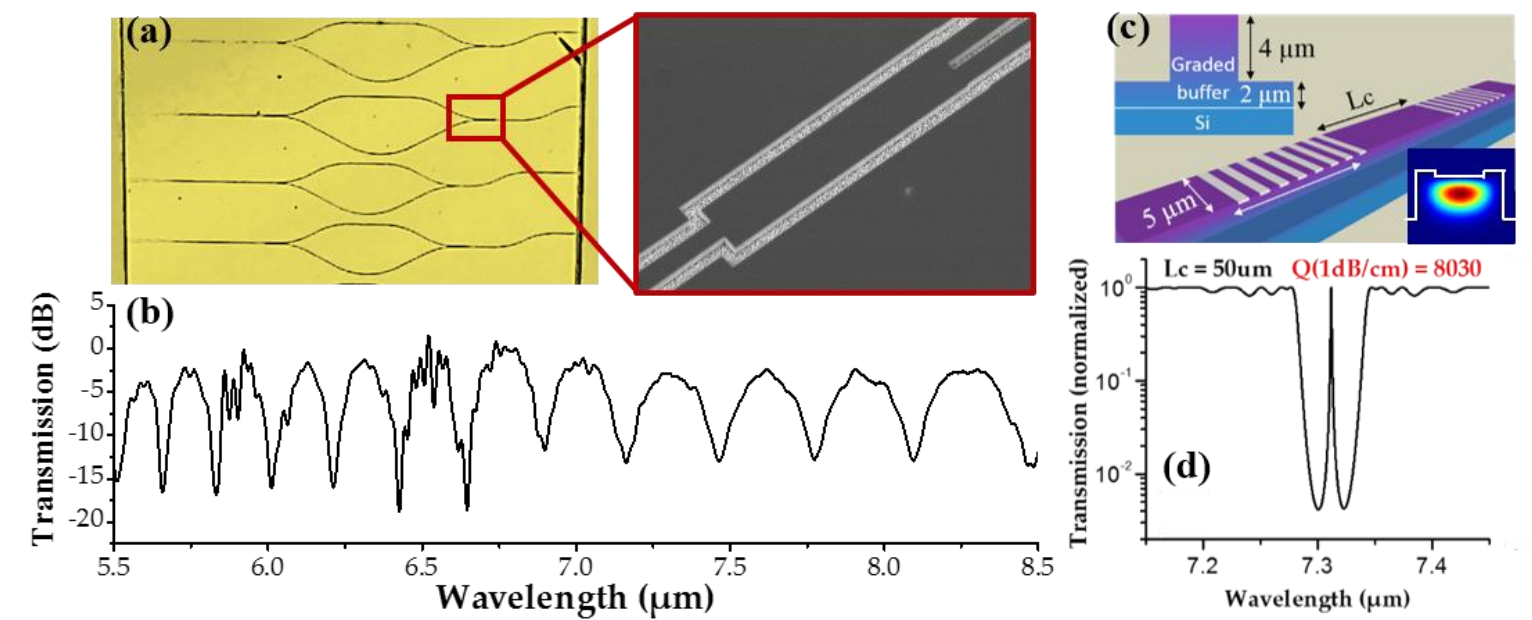

Figure 3. (a) Top microscope view of the fabricated asymmetric Mach-Zehnder interferometers with a zoomed-in region showing an SEM image of the multimode interferometer. (b) Measured spectral response of a Mach-Zehnder device with $\Delta L$ $=48 \mu \mathrm{m}$.

\section{NEW WAVEGUIDE DESIGNS FOR SUPERCONTINUUM GENERATION IN THE MID-IR}

In addition to the above-described passive mid-IR structures, there is also an increasing interest in developing mid-IR active devices able to exploit the nonlinearities of the raw materials. In particular, Ge-rich SiGe alloys are good candidates for that purpose since they show remarkably high third-order nonlinearity compared to other CMOS-compatible materials such as silicon oxide, silicon nitride or silicon. Moreover, these nonlinear effects become more efficient in Ge-based materials at longer wavelengths due to the suppression of two-photon absorption from $\lambda=3.2 \mu \mathrm{m}$ on. Consequently, any pumping signal with a wavelength centred beyond this value will potentially produce strong nonlinear effects in our Ge-rich SiGe platform provided that a proper waveguide dispersion engineering is accomplished. In order to fulfil this condition, an optimization of the waveguide geometry was carried out for a broad wavelength range from $\lambda=3 \mu \mathrm{m}$ to $8 \mu \mathrm{m}$. In this case, instead of using the waveguides previously described in figure 1(b), we have employed a new waveguide cross-section that consists in a single SiGe graded buffer with a linear increase of the Ge concentration from $\mathrm{Si}$ to pure $\mathrm{Ge}$ in a total thickness of $6 \mu \mathrm{m}$. Thus, the optical mode is now confined in the graded buffer directly. Particularly, figure 4(a) shows the new waveguide cross-section with the dispersion engineered geometric parameters, having a waveguide width of $\mathrm{W}=4 \mu \mathrm{m}$ and an etching depth of $\mathrm{D}=4 \mu \mathrm{m}$. These values were chosen based on a systematic study of the dispersion value as a function of both $\mathrm{W}$ and $\mathrm{D}$ for different wavelengths, as observed in the set of coloured panels on top of figure 4(b). It is worth to notice that by choosing the selected parameters (marked by a grey dot in each panel), flat anomalous dispersion enclosed in the low dispersion regime, i. e. D < $100 \mathrm{ps} / \mathrm{nm} / \mathrm{km}$, is obtained over one octave spanning for both polarizations, as illustrated in figure 4(c). The origin of such broadband characteristic is believed to be due to the gradual mode-size accommodation that takes place in graded SiGe waveguides as illustrated at the bottom of figure 4(b). As seen, the mode is confined in the upper part of the waveguide corresponding to the Ge-rich area, and provides a gradual downwards expansion of the propagating optical mode for longer operating wavelengths, delivering a unique dispersion compensation at each wavelength that can be exploited to develop efficient supercontinuum light sources in the mid-IR range. Moreover, a promising third-order nonlinear parameter is anticipated using this approach [9]. 
(a)

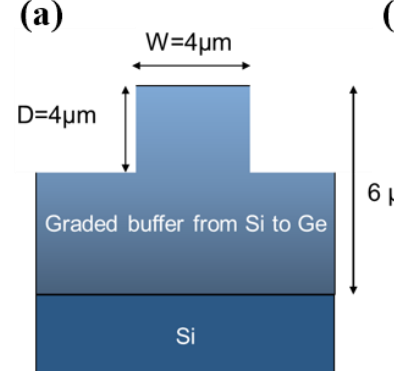

(b)

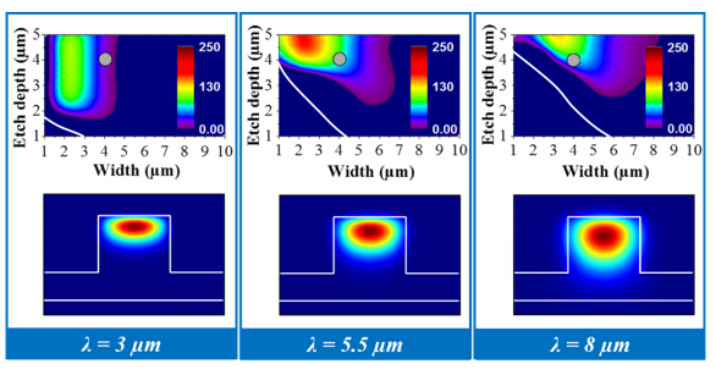

(c)

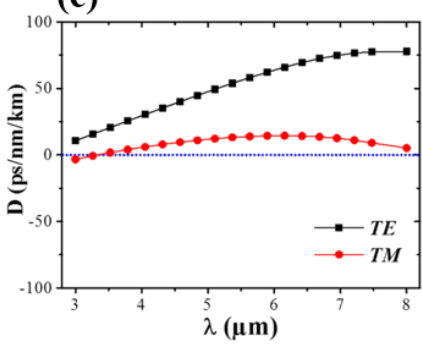

Figure 4. (a) Waveguide cross-section for the implementation of nonlinear devices. (b) (Top panels) Coloured maps showing the anomalous dispersion characteristic as a function of the waveguide geometry and the operating wavelength. (Bottom panels) Optical mode simulations as a function of the operating wavelength (TE polarization). (c) Calculated dispersion curves for a wavelength span of $\lambda=3-8 \mu \mathrm{m}$ for both TE (black squares) and TM (red dots) polarizations.

\section{CONCLUSIONS}

In conclusion, we have shown the recent advances on the implementation of a new mid-IR photonic integrated platform based on Ge-rich SiGe alloys. Progress towards the demonstration of several fundamental building blocks having broadband operation in the mid-IR has been revisited, including waveguides, MachZehnder interferometers, and nonlinear waveguides for supercontinuum generation. Moreover, a representative proof-of-concept that exploits the evanescent-field absorption in SiGe waveguides to retrieve several mid-IR absorption patterns of a standalone photoresist has been performed, demonstrating the potential of this platform for mid-IR photonic chip-scale sensors.

\section{ACKNOWLEDGEMENTS}

This project has received funding from the European Research Council (ERC) under the European Union's Horizon 2020 research and innovation program (grant agreement $\mathrm{N}^{\circ} 639107-$ INsPIRE). The fabrication of the devices was performed at the Plateforme de Micro-Nano-Technologie/C2N, which is partially funded by the "Conseil Général de l'Essonne". This work was partly supported by the French RENATECH network.

\section{REFERENCES}

[1] Richard Soref, "Mid-infrared photonics in silicon and germanium,"Nat. Photonics, 4(8) 495, 2010.

[2] A. Malik, et al. "Germanium-on-silicon mid-infrared arrayed waveguide grating multiplexers," IEEE Phot.Technol. Lett. 25(18), 1805 (2013).

[3] Z. Cheng, et al. "Mid-infrared suspended membrane waveguide and ring resonator on silicon-on-insulator," IEEE photonics journal, 4(5), 1510 (2012).

[4] M. Brun, et al. "Low loss SiGe graded index waveguides for mid-IR applications," Opt. express, 22(1), 508 (2014).

[5] J. M. Ramirez, et al. "Ge-rich SiGe waveguides for mid-infrared photonics," Proc. Of SPIE 10108 (2017).

[6] J. M. Ramirez, et al. "Low-loss Ge-rich Si 0.2 Ge 0.8 waveguides for mid-infrared photonics," Opt. Lett. 42 (1), 105 (2017).

[7] V. Vakarin et al, "Ultra-wideband Ge-rich silicon germanium integrated Mach Zehnder interferometer for mid infrared spectroscopy,” Opt. Lett. 42(17) 3482, 2017.

[8] F De Leonardis, et al. "Germanium-on-Silicon Waveguide Engineering for Third Harmonic Generation in the MidInfrared,” J. Lightwav. Technol. 33 (24), 5103, 2015.

[9] J. M. Ramirez et al, "Ge-rich graded-index Si1-xGex waveguides with broadband tight mode confinement and flat anomalous dispersion for nonlinear mid-infrared photonics," Opt. Express 25(6) 6561, 2017.

[10] S. Serna, et al. "Nonlinear Properties of Ge-rich Si 1-x Ge x Materials with Different Ge Concentrations," Sci. Rep. 7(1), 14692 (2017).

[11] J. M. Ramirez et al. "Graded SiGe waveguides with broadband low-loss propagation in the mid infrared," Opt. Express, 26(2) 870, 2018.

[12] Q. Liu et al. "Mid-infrared sensing between 5.2 and $6.6 \mu \mathrm{m}$ wavelength using Ge-rich SiGe waveguides," accepted in Opt. Mat. Express. 